Indian J. Pure Appl. Math., 47(4): 641-653, December 2016

(c) Indian National Science Academy

DOI: $10.1007 / \mathrm{s} 13226-016-0197-0$

\title{
KINEMATICAL CONSERVATION LAWS IN A SPACE OF ARBITRARY DIMENSIONS
}

\author{
Phoolan Prasad \\ Department of Mathematics, Indian Institute of Science, \\ Bangalore 560 012, India \\ e-mail: prasad@math.iisc.ernet.in
}

(Received 15 July 2015; after final revision 27 January 2016;

accepted 1 March 2016)

\begin{abstract}
In a large number of physical phenomena, we find propagating surfaces which need mathematical treatment. In this paper, we present the theory of kinematical conservation laws $(\mathrm{KCL})$ in a space of arbitrary dimensions, i.e., $d$-D KCL, which are equations of evolution of a moving surface $\Omega_{t}$ in $d$-dimensional $\boldsymbol{x}$-space, where $\boldsymbol{x}=\left(x_{1}, x_{2}, \ldots, x_{d}\right) \in \mathbb{R}^{d}$. The KCL are derived in a specially defined ray coordinates $\left(\boldsymbol{\xi}=\left(\xi_{1}, \xi_{2}, \ldots, \xi_{d-1}\right), t\right)$, where $\xi_{1}, \xi_{2}, \ldots, \xi_{d-1}$ are surface coordinates on $\Omega_{t}$ and $t$ is time. KCL are the most general equations in conservation form, governing the evolution of $\Omega_{t}$ with physically realistic singularities. A very special type of singularity is a kink, which is a point on $\Omega_{t}$ when $\Omega_{t}$ is a curve in $\mathbb{R}^{2}$ and is a curve on $\Omega_{t}$ when it is a surface in $\mathbb{R}^{3}$. Across a kink the normal $\boldsymbol{n}$ to $\Omega_{t}$ and normal velocity $m$ on $\Omega_{t}$ are discontinuous.
\end{abstract}

Key words : Kinematical conservation laws (KCL); Ray theory; conservation laws; propagation of a surface.

\section{INTRODUCTION}

In this paper, we study equations of evolution of a $(d-1)$ dimensional surface in $\boldsymbol{x}=$ $\left(x_{1}, x_{2}, \ldots, x_{d}\right)$ space. The surface occupies different positions at different times and is denoted by $\Omega_{t}$. For evolution of $\Omega_{t}$ we need to know its ray velocity $\chi$ which carries different points of the surface and gives rays. Once the ray velocity is known, we can easily write an eikonal equation of the surface (as shown below), which is a Hamilton-Jacobi PDE. There is now a standard theory to study viscosity solution of this first order PDE $[11,18]$, where the folded trailing part of $\Omega_{t}$, if formed by ray theory, disappears. However, when the rays have built in them effect of genuine nonlinearity of the equations governing a medium in which $\Omega_{t}$ propagates, a new type of singularity, called kink, appears on $\Omega_{t}$ [15]. Both the ray theory and the level set theory of [18] are inadequate to study formation and evolution of a kink. 
Distinguishing feature of a kink is in appearance of a discontinuity in the normal direction $\boldsymbol{n}$ and the velocity $m$ of $\Omega_{t}$ on the kink. The governing PDEs of $\Omega_{t}$, i.e., the eikonal equation and ray theory breakdown. Examples of $\Omega_{t}$ with discontinuities in $\boldsymbol{n}$ and $m$ across a $d-2$ dimensional surface on $\Omega_{t}$ are plenty. The first theoretical evidence is in Whitham's 1957 and 1959 work (see [21]) on shock propagation, where he called the point of discontinuity on a shock front in 2-D and the curve of discontinuity on a shock front in 3-D 'shock-shock'. Experimental evidence of such a discontinuity on a shock front in gasdynamics was first shown by Sturtevant and Kulkarni in 1976 [19]. In a numerical computation of successive positions of a shock front by a shock ray theory [17], Kevlhan [13] studied in 1996 the formation and propagation of shock-shocks by a special method, which is difficult to continue for a long time. In order to study formation and evolution of kinks on $\Omega_{t}$ and detailed evolution of the surface itself, we developed kinematical conservation laws (KCL) in two and three space dimensions respectively in 1992 [14]; and 1996 [12], 2009 [1] and 2010 [3] in ray coordinate systems. Since these discontinuities can appear not only on a shock front but on any moving surface $\Omega_{t}$, we call them kinks. The 2-D KCL and 3-D KCL have been extensively used to solve many practical problems, see $[2,4,5,6,7,8,9]$ and the older references in these. Hence, it is worth developing the KCL theory for a space of arbitrary dimensions for a mathematical completeness. There is also an opportunity to extend KCL theory to non-Euclidean spaces and study evolution of surfaces there (e.g., in cosmology using general theory of relativity, where nonlinear waves appear very frequently [20]).

Ray coordinate system: As mentioned earlier, we consider evolution of a surface $\Omega_{t}$ in $d$-dimensional $\boldsymbol{x}$-space, where $\boldsymbol{x}=\left(x_{1}, x_{2}, \ldots, x_{d}\right) \in \mathbb{R}^{d}$. Let $\boldsymbol{\xi}=\left(\xi_{1}, \xi_{2}, \cdots, \xi_{d-1}\right)$ be a set of surface coordinates on $\Omega_{t}$, these coordinates also evolve with time $t$. The surface $\Omega_{t}$ in $\boldsymbol{x}$-space is generated by a $d-2$ parameter family of curves such that along each of these curves a particular coordinate $\xi_{p}$ varies and the $d-2$ parameters $\xi_{1}, \cdots, \xi_{p-1}, \xi_{p+1}, \cdots, \xi_{d-1}$ are constant ${ }^{1}$. This is true for each index $p=1,2, \cdots, d-1$. Through any point $\boldsymbol{\xi}$ of $\Omega_{t}$ there passes a ray (in $\boldsymbol{x}$-space) associated with the evolution of $\Omega_{t}$.

Since $\Omega_{t}$ evolves with $t$, it is represented by an equation

$$
\Omega_{t}: \varphi(\boldsymbol{x}, t)=0, \quad t=\text { constant }
$$

The unit normal $\boldsymbol{n}$ and the normal velocity $m$ of $\Omega_{t}$ are given by

$$
\boldsymbol{n}=\nabla \varphi /|\nabla \varphi|, \quad m=-\varphi_{t} /|\nabla \varphi|
$$

\footnotetext{
${ }^{1} \mathrm{~A}$ repeatetion of a symbol $p$ and $q$ in subscript or superscript in a term will imply summation over the range $1,2, \cdots, d-1$.
} 
Let the ray velocity be $\boldsymbol{\chi}=\left(\chi_{1}, \chi_{2}, \cdots, \chi_{d}\right)$, then $m=\langle\boldsymbol{n}, \boldsymbol{\chi}\rangle$ and from (1.2) $\varphi$ satisfies the the eikonal equation

$$
\varphi_{t}+\langle\chi, \nabla \varphi\rangle=0
$$

The rays from different points of $\Omega_{t}$ are governed by ray equations [16] which are Charpit's equations $^{2}$ of the eikonal equation (1.3) and rays form a $d-1$ parameter family of curves in the physical space, i.e., the $\boldsymbol{x}$-space.

Given a time $t$, we know the position $\Omega_{t}$ of the moving surface and given $\boldsymbol{\xi}$ we get a unique point $\boldsymbol{x}$ on $\Omega_{t}$. Similarly, given a point $\boldsymbol{x} \in \mathbb{R}^{d}$, we know the time $t$ when $\Omega_{t}$ arrives here and there is a value of $\boldsymbol{\xi}$ at this point. We assume that the mapping between the ray coordinates $(\boldsymbol{\xi}, t)$ and the spatial coordinates $\boldsymbol{x}=\left(x_{1}, x_{2}, \cdots, x_{d}\right)$ is locally one-to-one. This is true as long as the $d-1$ tangent vectors of the coordinate curves are linearly independent. Note the $\boldsymbol{n}$ is transversal to $\Omega_{t}$. Thus, given a domain in the physical space we have two sets of coordinate systems, physical coordinates $\boldsymbol{x}$ and ray coordinates $(\boldsymbol{\xi}, t)$.

Metric associated with a coordinate $\xi_{p}$ and that with $t$ : Let the metric associated with the surface coordinate $\xi_{p}$ be $g_{p}$. Then $g_{p} d \xi_{p}$ (no sum over the repeated subscript here) is an element of distance along the coordinate line along which $\xi_{p}$ varies. The speed of a point moving along the ray with the ray velocity $\chi$ is $|\chi|$. Then, while moving with the the ray velocity, $|\chi| d t$ is the displacement along the ray in time $d t$. Thus $|\chi|$ is the metric associated with the coordinate $t$. When $\chi$ is in the direction of the normal to $\Omega_{t}$, as assumed below, the metric associated with $t$ is $m$.

\section{2. $d$-DIMENSIONAL KCL}

We consider here the case when the motion of the surface $\Omega_{t}$ is isotropic in the sense that the associated ray velocity $\boldsymbol{\chi}$ depends on the unit normal $\boldsymbol{n}$ by the relation

$$
\chi=m n
$$

where $m$ is the velocity of the surface ${ }^{3}$. The eikonal equation (1.3) becomes $\varphi_{t}+m|\nabla \varphi|=0$. In this case, the ray equations (2.4.6) and (2.4.7) of [15] or (1.6a) and (1.6b) of [16] take simple form

$$
\begin{gathered}
\frac{d \boldsymbol{x}}{d t}=m \boldsymbol{n},|\boldsymbol{n}|=1, \\
\frac{d \boldsymbol{n}}{d t}=-\boldsymbol{L} m:=-(\nabla-\boldsymbol{n}\langle\boldsymbol{n}, \nabla\rangle) m .
\end{gathered}
$$

\footnotetext{
${ }^{2}$ In most books, these equations have been simply called 'characteristic equations' and no credit has been given to Charpit.

${ }^{3}$ Velocity of a surface means the normal velocity of the surface.
} 
where, the operator $\boldsymbol{L}$ defined above represents differentiation in a direction tangential to the surface $\Omega_{t}$. Since $|\boldsymbol{n}|=1$, only $d-1$ equations in (2.3) are independent.

Let $\boldsymbol{u}_{1}, \boldsymbol{u}_{2}, \cdots, \boldsymbol{u}_{d-1}$ be unit vectors along the coordinates $\xi_{1}, \xi_{2}, \cdots, \xi_{d-1}$. Note that, like $\boldsymbol{n}, \boldsymbol{u}_{p} \in \mathbb{R}^{d}$. The unit vector along the ray, i.e., $\boldsymbol{n}$ is in the direction normal to the surface $\Omega_{t}$ and hence $\left\langle\boldsymbol{u}_{p}, \boldsymbol{n}\right\rangle=0$.

Increments $d \xi_{1}, d \xi_{2}, \cdots, d \xi_{d-1}$ and $d t$ in the ray coordinates leads to a displacement $d \boldsymbol{x}$ in $\boldsymbol{x}$-space given by

$$
d \boldsymbol{x}=\left(g_{1} \boldsymbol{u}_{1}\right) d \xi_{1}+\left(g_{2} \boldsymbol{u}_{2}\right) d \xi_{2}+\cdots+\left(g_{d-1} \boldsymbol{u}_{d-1}\right) d \xi_{d-1}+(m \boldsymbol{n}) d t .
$$

This gives $\boldsymbol{x}_{\xi_{p}}=g_{p} \boldsymbol{u}_{p}$ and $\boldsymbol{x}_{t}=m \boldsymbol{n}$. For a smooth moving surface $\Omega_{t}$, we equate $\boldsymbol{x}_{\xi_{p} t}=\boldsymbol{x}_{t \xi_{p}}$ and get the $d$-D KCL system

$$
\left(g_{p} \boldsymbol{u}_{p}\right)_{t}-(m \boldsymbol{n})_{\xi_{p}}=0, \text { no sum over } p .
$$

For $p=1,2, \cdots, d-1$, the KCL system is of $d-1$ vector equations, each one with $d$ components.

We also equate $\boldsymbol{x}_{\xi_{p} \xi_{q}}$ and $\boldsymbol{x}_{\xi_{q} \xi_{p}}$ to derive $(d-2)$ ! more vector equations:

$$
\left(g_{q} \boldsymbol{u}_{q}\right)_{\xi_{p}}-\left(g_{p} \boldsymbol{u}_{p}\right)_{\xi_{q}}=0 \text {, no sum over repeated subscripts. }
$$

Theorem 2.1 - For a smooth $\Omega_{t}$ equations (2.5) imply that $\left(g_{q} \boldsymbol{u}_{q}\right)_{\xi_{p}}-\left(g_{p} \boldsymbol{u}_{p}\right)_{\xi_{q}}$ is independent of $t$.

Proof : Differentiate (2.5) with respect to $\xi_{q}$ and (2.5) with $p$ replaced by $q$ with respect to $\xi_{p}$ and subtract the second result from the first to get

$$
\left(\left(g_{q} \boldsymbol{u}_{q}\right)_{\xi_{p}}-\left(g_{p} \boldsymbol{u}_{p}\right)_{\xi_{q}}\right)_{t}=0 \text {, no sum over repeated subscripts. }
$$

This completes the proof of the theorem.

When coordinates $\xi_{1}, \xi_{2}, \cdots, \xi_{d-1}$ are chosen on $\Omega_{0}$, i.e. at $t=0$, the expression for a displacement $d \boldsymbol{x}$ on $\Omega_{0}$ will be given by (2.4) with $d t=0$. Hence the conditions (2.6) will be automatically satisfied at $t=0$. The theorem implies that the equations (2.6) are satisfied at all $t>0$. The KCL consists of just $d(d-1)$ conservation laws.

The equations (2.6) simply appear as constraints, just as solenoidal conditions in the equations of magnetohydrodynamics. Hence we call the equations (2.6) as geometrical solenoidal constraint.

Remark 2.2 : Once we have chosen the coordinates $\boldsymbol{\xi}=\left(\xi_{1}, \xi_{2}, \cdots, \xi_{d-1}\right)$ arbitrarily on $\Omega_{0}$, they remain coordinates on $\Omega_{t}, \forall t>0$ as if they are glued to it. Their directions $\boldsymbol{u}_{1}, \boldsymbol{u}_{2}, \cdots$, 
$\boldsymbol{u}_{d-1}$ evolve with time as the surface $\Omega_{t}$ evolves. We can initially choose an orthogonal coordinate system on $\Omega_{0}$ but for $t>0$ the system, in almost all cases, will cease to be orthogonal $^{4}$.

KCL system is under-determined: We first note that the unit vector $\boldsymbol{n}$, which has $d$ components, satisfies $d-1$ linear homogeneous equations $\left\langle\boldsymbol{u}_{p}, \boldsymbol{n}\right\rangle=0, p=1,2, \cdots, d-1$. Hence, $\boldsymbol{n}$ can be expressed uniquely in terms of the $d-1$ vectors $\boldsymbol{u}_{p}$. Therefore, the unknowns appearing in the system (2.5) are:

- $g_{1}, g_{2}, \cdots, g_{d-1}$, which are $d-1$,

- $m$, which is just one and

- $\boldsymbol{u}_{1}, \boldsymbol{u}_{2}, \cdots, \boldsymbol{u}_{d-1}$, have $(d-1)^{2}$ independent quantities since $\left|\boldsymbol{u}_{p}\right|=1$.

Thus we have $d(d-1)+1$ unknowns in $d(d-1)$ equations $(2.5)$ and hence the KCL system is under-determined. This is expected, as we have taken a propagating front $\Omega_{t}$ without prescribing the physical nature of the front. The system can be closed only with the help of additional relations or equations, which would follow from the nature of the surface $\Omega_{t}$, i.e., the dynamics of the medium in which it propagates.

Differential form of KCL: Now we derive the differential form of the KCL system (2.5) assuming that the functions $\boldsymbol{u}_{p}, \boldsymbol{n}, m$ and $g_{p}$, are smooth. We first note that $\left\langle\boldsymbol{u}_{p}, \boldsymbol{n}\right\rangle=0$ implies $\left\langle\boldsymbol{u}_{p}, \boldsymbol{n}_{\xi_{q}}\right\rangle=-\left\langle\left(\boldsymbol{u}_{p}\right)_{\xi_{q}}, \boldsymbol{n}\right\rangle$. Further, since $\left|\boldsymbol{u}_{p}\right|=1$, we have $\left\langle\boldsymbol{u}_{p},\left(\boldsymbol{u}_{p}\right)_{t}\right\rangle=0$. Writing the differential form of $(2.5)$ and taking inner product with $\boldsymbol{u}_{p}$, we get

$$
\left(g_{p}\right)_{t}=-m\left\langle\boldsymbol{n},\left(\boldsymbol{u}_{p}\right)_{\xi_{p}}\right\rangle \text {, no sum over } p .
$$

In the differential form of (2.5), we now use the expression for $\left(g_{p}\right)_{t}$ from $(2.7)$ to get

$$
g_{p}\left(\boldsymbol{u}_{p}\right)_{t}=m_{\xi_{p}} \boldsymbol{n}+m\left\langle\boldsymbol{n},\left(\boldsymbol{u}_{p}\right)_{\xi_{p}}\right\rangle \boldsymbol{u}_{p}+m \boldsymbol{n}_{\xi_{p}} \text {, no sum over } p .
$$

The total number of equations in (2.7) and (2.8) are $d+d(d-1)=d^{2}$, but we shall show that only $d(d-1)$ are independent. We prove a theorem:

Theorem 2.3 - For any given p, the last equation in the equation in (2.8) namely

$$
g_{p}\left(u_{p d}\right)_{t}=m_{\xi_{p}} n_{d}+m\left\langle\boldsymbol{n},\left(\boldsymbol{u}_{p}\right)_{\xi_{p}}\right\rangle u_{p d}+m\left(n_{d}\right)_{\xi_{p}}, \text { no sum over } p
$$

\footnotetext{
${ }^{4}$ Let me first give three cases of $\Omega_{t}$, when orthogonality is not lost: (1) plane, (ii) a circular cylinder and (iii) a sphere, all three with suitable choices surface coordinates and with constant distributions of $m$ at $t=0$. I do not know if such cases form a set of measure zero in the set of all $\Omega_{t}$ but the statement 'almost all' seems to be appropriate. If the orthogonality is lost, it becomes very difficult to find the eigenvalues and discuss the nature of the eigenspace of a system of equations in KCL based applications as one can see in references [1] and [3], which deal with $\Omega_{t}$ in $3-\mathrm{D}$.
} 
follow from its first d-1 equations

$$
g_{p}\left(u_{p q}\right)_{t}=m_{\xi_{p}} n_{q}+m\left\langle\boldsymbol{n},\left(\boldsymbol{u}_{p}\right)_{\xi_{p}}\right\rangle u_{p q}+m\left(n_{q}\right)_{\xi_{p}} \text {, no sum over } p ; q=1,2, \cdots, d-1 .
$$

Proof : We multiply (2.10) by $u_{p q}$ and sum over $q$ on the range $1,2, \cdots, d-1$ to get

$$
\begin{array}{r}
\frac{1}{2} g_{p}\left\{\left(u_{p 1}\right)^{2}+\left(u_{p 2}\right)^{2}+\cdots+\left(u_{p(d-1)}\right)^{2}\right\}_{t}=m_{\xi_{p}}\left\{n_{1} u_{p 1}+n_{2} u_{p 2}+\cdots+n_{(d-1)} u_{p(d-1)}\right\} \\
+m\left\langle\boldsymbol{n},\left(\boldsymbol{u}_{p}\right)_{\xi_{p}}\right\rangle\left\{u_{p 1}^{2}+u_{p 2}^{2}+\cdots+u_{p(d-1)}^{2}\right\}+m\left\{u_{p 1}\left(n_{1}\right)_{\xi_{p}}+u_{p 2}\left(n_{2}\right)_{\xi_{p}}+\cdots\right. \\
\left.+u_{p(d-1)}\left(n_{d-1}\right)_{\xi_{p}}\right\}, \text { no sum over } p .
\end{array}
$$

Now we use, without summation convention,

$$
\begin{aligned}
& \left(u_{p 1}\right)^{2}+\left(u_{p 2}\right)^{2}+\cdots+\left(u_{p(d-1)}\right)^{2}=1-\left(u_{p d}\right)^{2} \\
& n_{1} u_{p 1}+n_{2} u_{p 2}+\cdots+n_{(d-1)} u_{p(d-1)}=-n_{d} u_{p d} \\
& u_{p 1}\left(n_{1}\right)_{\xi_{p}}+u_{p 2}\left(n_{2}\right)_{\xi_{p}}+\cdots+u_{p(d-1)}\left(n_{d-1}\right)_{\xi_{p}} \\
& \quad=\left\langle\boldsymbol{u}_{p}, \boldsymbol{n}_{\xi_{p}}\right\rangle-u_{p d}\left(n_{d}\right)_{\xi_{p}}=-\left\langle\left(\boldsymbol{u}_{p}\right)_{\xi_{p}}, \boldsymbol{n}\right\rangle-u_{p d}\left(n_{d}\right)_{\xi_{p}}
\end{aligned}
$$

and divide the resulting equation by $u_{p d}$ to get (2.9).

The theorem is now proved.

We express $u_{1 d}$ in terms of $u_{11}, u_{12}, \cdots, u_{1(d-1)}$ using $u_{1 d}^{2}=1-\left(u_{11}^{2}+u_{12}^{2}+\cdots+u_{1(d-1)}^{2}\right)$ and do this for the last components of rest of the vectors $\boldsymbol{u}_{2}, \cdots, \boldsymbol{u}_{(d-1)}$. Then we have expressed $u_{1 d}, u_{2 d}, \cdots, u_{(d-1) d}$ in terms of $u_{p 1}, u_{p 2}, \cdots, u_{p(d-1)} ; p=1,2, \cdots, d-1$. Finally, the equations (2.7) and (2.10) are a set of $d(d-1)$ independent differential form of the KCL system containing the $d(d-1)+1$ quantities $m ; g_{p} ; u_{p 1}, u_{p 2}, \cdots, u_{p(d-1)} ; p=1,2, \cdots, d-1$.

\section{Equivalence of KCL and Ray Equations}

The equivalence of the KCL system for smooth solutions and the ray equations is not surprising as both are related geometrically. This equivalence is to be shown between ray equations (2.2) and (2.3); and the differential forms (2.7) and (2.8) of the KCL. In this section there is no sum over $p$.

Let us first give an explicit derivation of the KCL system from the ray equations.

Derivation of (2.7): (2.4) gives $\boldsymbol{x}_{\xi_{p}}=g_{p} \boldsymbol{u}_{p}$ which implies $g_{p}^{2}=x_{1} \underset{\xi_{p}}{2}+x_{2} \underset{\xi_{p}}{2}+\cdots+x_{d_{\xi_{p}}}^{2}$ $=\left|\boldsymbol{x}_{\xi_{p}}\right|^{2}$. Differentiating it with respect to $t$, using $\boldsymbol{x}_{\xi_{p} t}=\boldsymbol{x}_{t \xi_{p}} \equiv\left(\boldsymbol{x}_{t}\right)_{\xi_{p}}, \boldsymbol{x}_{\xi_{p}}=g_{p} \boldsymbol{u}_{p}$ and dividing by $g_{p}$ we get

$$
g_{p_{t}}=\left\langle\boldsymbol{u}_{p},\left(\boldsymbol{x}_{t}\right)_{\xi_{p}}\right\rangle .
$$


Using (2.5) we derive

$$
g_{p_{t}}=\left\langle\boldsymbol{u}_{p}, m_{\xi_{p}} \boldsymbol{n}+m \boldsymbol{n}_{\xi_{p}}\right\rangle=\left\langle\boldsymbol{u}_{p}, m \boldsymbol{n}_{\xi_{p}}\right\rangle=-m\left\langle\boldsymbol{n},\left(\boldsymbol{u}_{p}\right)_{\xi_{p}}\right\rangle
$$

which is the equation (2.7).

Derivation of (2.8): We differentiate with respect to $t$ the relation $g_{p} \boldsymbol{u}_{p}=\boldsymbol{x}_{\xi_{p}}$, and use $\boldsymbol{x}_{\xi_{p} t}=\boldsymbol{x}_{t \xi_{p}}=(m \boldsymbol{n})_{\xi_{p}}$ and $g_{p_{t}}=-m\left\langle\boldsymbol{n},\left(\boldsymbol{u}_{p}\right)_{\xi_{p}}\right\rangle$ to give

$$
g_{p}\left(\boldsymbol{u}_{p}\right)_{t}=m\left\langle\boldsymbol{n},\left(\boldsymbol{u}_{p}\right)_{\xi_{p}}\right\rangle \boldsymbol{u}_{p}+\boldsymbol{n} m_{\xi_{p}}+m \boldsymbol{n}_{\xi_{p}},
$$

which is the equation (2.8).

An alternative proof: Next we give a geometrical proof, which is beautiful and equally simple. Let $m$ be a smooth function of $\boldsymbol{x}$ and $t$ and let $\boldsymbol{x}, \boldsymbol{n}$ (with $|\boldsymbol{n}|=1$ ) satisfy the ray equations (2.2) and (2.3), which give successive positions of a moving surface $\Omega_{t}$. Choose a coordinate system $\xi_{1}, \xi_{2}, \cdots, \xi_{p-1}$ on $\Omega_{t}$ with metrics $g_{1}, g_{2}, \cdots, g_{p-1}$ associated with these coordinates. Let $\left(\boldsymbol{u}_{1}, \boldsymbol{u}_{2}, \cdots, \boldsymbol{u}_{p-1}\right)$ be unit tangent vectors along the coordinates curves. Then the derivation given in the beginning of the last section leads to the $d$-D KCL system (2.5) along with the geometrical solenoidal constraint (2.6). Thus the ray equations imply $d$-D KCL.

KCL implies ray equations: We take $d$ smooth linearly independent unit vector fields $\boldsymbol{u}_{1}, \boldsymbol{u}_{2}, \cdots, \boldsymbol{u}_{d-1}, \boldsymbol{n}$ and $d$ smooth scalar functions $g_{1}, g_{2}, \cdots, g_{d-1}, m$ in $\left(\xi_{1}, \xi_{2}, \cdots, \xi_{d-1}, t\right)-$ space such that $\boldsymbol{n}$ is orthogonal to each of $\boldsymbol{u}_{1}, \boldsymbol{u}_{2}, \cdots, \boldsymbol{u}_{d-1}$; and they satisfy the KCL (2.5) and the geometrical solenoidal constraint (2.6).

According to the fundamental integrability theorem ([10]-page 104), the conditions (2.5) and (2.6) imply the existence of a vector $\boldsymbol{x}$ satisfying

$$
\left(\boldsymbol{x}_{t}, \boldsymbol{x}_{\xi_{1}}, \cdots, \boldsymbol{x}_{\xi_{d-1}}\right)=\left(m \boldsymbol{n}, g_{1} \boldsymbol{u}_{1}, \cdots, g_{d-1} \boldsymbol{u}_{d-1}\right)
$$

Since the vectors on the right hand side are linearly independent, this gives a local one to one mapping between $\boldsymbol{x}$-space and $(\boldsymbol{\xi}, t)$-space. Let the plane $t=$ constant in $(\boldsymbol{\xi}, t)$-space be mapped on to a surface $\Omega_{t}$ in $\boldsymbol{x}$-space on which $\xi_{1}, \xi_{2}, \cdots, \xi_{p-1}$ are surface coordinates. Then $\boldsymbol{u}_{1}, \boldsymbol{u}_{2}, \cdots, \boldsymbol{u}_{p-1}$ are tangent vectors to $\Omega_{t}$ and $\boldsymbol{n}$ is orthogonal to $\Omega_{t}$, i.e., it is unit normal vector of $\Omega_{t}$. Let $\varphi(\boldsymbol{x}, t)=0$ be the equation of $\Omega_{t}$, then $\boldsymbol{n}=\nabla \varphi /|\nabla \varphi|$. The relation $\boldsymbol{x}_{t}=m \boldsymbol{n}$ in (3.4) is nothing but the first part (2.2) of the ray equations. The function $\varphi$ now satisfies the eikonal equation $\varphi_{t}+m|\nabla \varphi|=0$, which implies (2.3), see also [16]. Thus, we have derived the ray equations from KCL.

Now we have completed the proof of the theorem: 
Theorem 3.1 - For a given smooth function $m$ of $\boldsymbol{x}$ and $t$, the ray equations (2.2) and (2.3) are equivalent to the KCL as long as their solutions are smooth.

\section{Kink Phenomenon}

In derivation of KCL and discussion of some of its properties, we have taken the functions appearing in the equations to be smooth and the transformation between the ray coordinates $(\boldsymbol{\xi}, t)$ and physical coordinates $\boldsymbol{x}$ be nonsingular. When a singularity in the transformation appears, the shape of a surface $\Omega_{t}$ would become very complex in multi-dimensions, and probably no complete theory is available. A very brief discussion of it is available in section 3.3 .3 of [15]. In this section we devote to images on $\boldsymbol{x}$-space of a special type of singularity in functions in the ray coordinates.

The system (2.5) consists of equations which are conservation laws, so its weak solution may contain a shock surface represented $\mathcal{S}$, which is a $d$-1 dimensional surface in $(\boldsymbol{\xi}, t)$ space $^{5}$. Across this shock surface the $d$ scalars $m, g_{p}$ and $d-1$ vectors $\boldsymbol{u}_{\boldsymbol{p}}$; and $\boldsymbol{n}$ will be discontinuous and will satisfy Rankine-Hugoniot relations. Image of a shock surface $\mathcal{S}$ into $\boldsymbol{x}$-space will be another $d$-1 dimensional surface, let us call it a kink surface and denote it by $\mathcal{K}$. The surface $\mathcal{K}$ will intersect $\Omega_{t}$ in a $d-2$ dimensional surface, say kink which we denote by $\mathcal{K}_{t}$. In order to avoid using 'kink surface' for both $\mathcal{K}$ and $\mathcal{K}_{t}$, we call the later one simply 'kink'. In 2-D a kink becomes a point (Figure 1), in 3-D it becomes a curve (Figure 2) and in $d$-D it is a $d-2$ dimensional surface. As time $t$ evolves, $\mathcal{K}_{t}$ will generate the kink surface $\mathcal{K}$. When a ray (which is a curve) crosses the kink surface $\mathcal{K}$ at time $t$, the direction of the ray or normal direction $\boldsymbol{n}$ of $\Omega_{t}$ jumps as seen in the Figure 1. We assume that the mapping between $(\boldsymbol{\xi}, t)$-space and $\boldsymbol{x}$-space continues to be one to one even when a kink appears.

Rankine-hugoniot $(\mathbf{R H})$ relations and conservation of distance: Consider any two points $P^{\prime}(\boldsymbol{x})$ and $Q(\boldsymbol{x}+d \boldsymbol{x})$ in the physical space corresponding to points $(\boldsymbol{\xi}, t+d t)$ and $(\boldsymbol{\xi}+d \boldsymbol{\xi}, t)$ in the ray coordinate space. The distance between $P^{\prime}$ and $Q$ is given by (2.4). Let us assume that $P^{\prime}$ and $Q$ lie on the surfaces $\Omega_{t+d t}$ and $\Omega_{t}$ respectively and we further assume that they lie on a kink surface $\mathcal{K}$, which intersects $\Omega_{t}$ and $\Omega_{t+d t}$ respectively in kinks $\mathcal{K}_{t}$ and $\mathcal{K}_{t+d t}$. Now $(\boldsymbol{\xi}, t+d t)$ and $(\boldsymbol{\xi}+d \boldsymbol{\xi}, t)$ lie on a shock surface $\mathcal{S}$. In Figure 1 , we present a $2-\mathrm{D}$ analog of this situation.

We express the segment $Q P^{\prime}=d \boldsymbol{x}$ in terms of the quantities in the states on the negative and positive sides of the $\mathcal{K}$ denoted by subscripts $\ell$ and $r$ respectively. The conservation of $d \boldsymbol{x}$ implies that the expression for $(d \boldsymbol{x})_{\ell}$ on one side of the kink surface must be equal to that

\footnotetext{
${ }^{5} \mathrm{~A}$ shock front (a phrase very commonly used in literature) is a $d-2$ dimensional surface $\mathcal{S}_{t}$ in $d$-1 dimensional -space and its motion as $t$ varies generates the shock surface $\mathcal{S}$ in $(, t)$-space.
} 


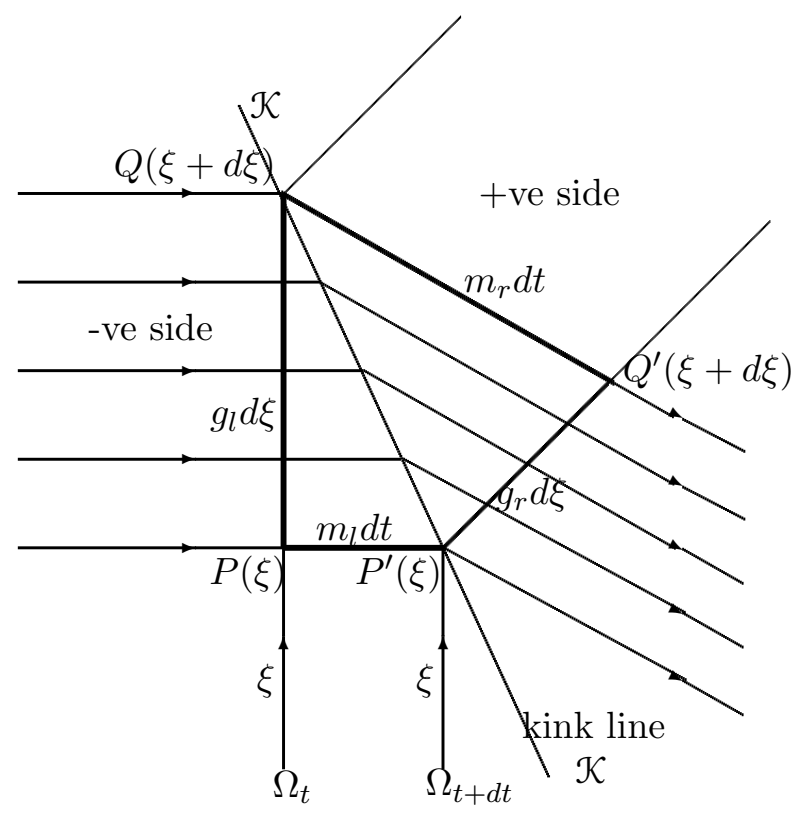

Figure 1: A 2-D analog of the kink phenomenon in $\left(x_{1}, x_{2}\right)$-plane corresponding to a shock moving down on $\Omega_{t}$ in negative $\xi$-direction, here $d \xi>0$. In this case $\Omega_{t}$ is a curve consisting of two lines meeting at a kink $\mathcal{K}_{t}$, which is a point $Q$ on the ray corresponding to $\xi+d \xi$. $\Omega_{t+d t}$ is a curve with kink at $P^{\prime}$ on the ray corresponding to $\xi$. The kink surface $\mathcal{K}$ becomes a kink line passing through the points $Q$ and $P^{\prime}$.

for $(d \boldsymbol{x})_{r}$, i.e.,

$$
\begin{aligned}
d \boldsymbol{x} & =\left(g_{1 \ell} \boldsymbol{u}_{1 \ell}\right) d \xi_{1}+\left(g_{2 \ell} \boldsymbol{u}_{2 \ell}\right) d \xi_{2}+\cdots+\left(g_{(d-1) \ell} \boldsymbol{u}_{(d-1) \ell}\right) d \xi_{d-1}+\left(m_{\ell} \boldsymbol{n}_{\ell}\right) d t \\
& =\left(g_{1 r} \boldsymbol{u}_{1 r}\right) d \xi_{1}+\left(g_{2 r} \boldsymbol{u}_{2 r}\right) d \xi_{2}+\cdots+\left(g_{(d-1) r} \boldsymbol{u}_{(d-1) r}\right) d \xi_{d-1}+\left(m_{r} \boldsymbol{n}_{r}\right) d t
\end{aligned}
$$

We have assumed $Q P^{\prime}=d \boldsymbol{x}$ on the kink surface $\mathcal{K}$ and its image $(d \boldsymbol{\xi}, d t)$ in ray coordinate space is on the shock surface $\mathcal{S}$. Projection of $(d \boldsymbol{\xi}, d t)$ on $\boldsymbol{\xi}$-space need not be in a direction normal to the shock front $\mathcal{S}_{t}$. We take the direction of the line element $Q P^{\prime}$ in $\boldsymbol{x}$-space such that this projection on $\boldsymbol{\xi}$-space is in the direction of the normal to the shock front in $\boldsymbol{\xi}$-space, then the differentials are further restricted. Let the unit normal of the shock front $\mathcal{S}_{t}$ be $\boldsymbol{E}=\left(E_{1}, E_{2}, \cdots, E_{d-1}\right)$ in $\boldsymbol{\xi}$-space and let the scalar $K$ be its velocity of propagation of $\mathcal{S}_{t}$ in this space, then the differentials in (4.1) satisfy $\frac{d \xi_{p}}{d t}=E_{p} K$ and (4.1) now becomes

$$
\begin{aligned}
& \left(g_{1 \ell} E_{1} \boldsymbol{u}_{1 \ell}+g_{2 \ell} E_{2} \boldsymbol{u}_{2 \ell}+\cdots+g_{(d-1) \ell} E_{d-1} \boldsymbol{u}_{(d-1) \ell}\right) K+m_{\ell} \boldsymbol{n}_{\ell} \\
& =\left(g_{1 r} E_{1} \boldsymbol{u}_{1 r}+g_{2 r} E_{2} \boldsymbol{u}_{2 r}+\cdots+g_{(d-1) r} E_{d-1} \boldsymbol{u}_{(d-1) r}\right) K+m_{r} \boldsymbol{n}_{r} .
\end{aligned}
$$




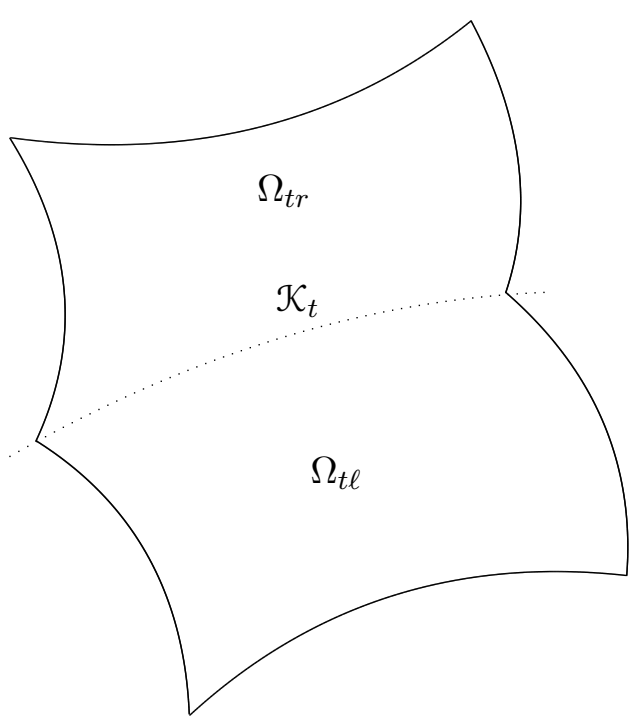

Figure 2: Figure is drawn in 3 space dimensions. Kink $\mathcal{K}_{t}$ (shown with dotted lines) on $\Omega_{t}=\Omega_{t r} \cup \Omega_{t \ell}$.

Remark 4.1 : From the discussion above it follows that (4.2) is a condition for the conservation of distance across a kink $\mathcal{K}_{t}$ when we choose $\frac{d \boldsymbol{\xi}}{d t}=\boldsymbol{E} K$. We note that each of $\boldsymbol{u}_{p}$ is a vector with $d$ components, therefore, the conservation of distance refers to conservation in all $d$ coordinate directions and hence in any direction in physical $\boldsymbol{x}$-space.

Using the usual method [11] for the derivation of jump conditions across a shock front, we deduce the following $\mathrm{RH}$ conditions from conservation laws (2.5)

$$
K\left[g_{p} \boldsymbol{u}_{p}\right]+E_{p}[m \boldsymbol{n}]=0, \text { no sum over } p ; \quad p=1,2, \cdots, d-1 \text {. }
$$

Multiplying (4.3) by $E_{p}$, summing over the subscript $p$ on its range $1,2, \cdots, d-1$ and using $|\boldsymbol{E}|=1$, we get

$$
K\left(E_{1}\left[g_{1} \boldsymbol{u}_{1}\right]+E_{1}\left[g_{1} \boldsymbol{u}_{1}\right]+\cdots+E_{p-1}\left[g_{p-1} \boldsymbol{u}_{p-1}\right]\right)+[m \boldsymbol{n}]=0,
$$

which is the same as (4.2). Thus we have proved extension of a theorem of GPR [12] for 3-D space.

Theorem 4.2 - The $d(d-1)$ jump relations (4.3) imply conservation of distance in $x_{1}, x_{2}, \cdots, x_{d}$ directions (and hence in any arbitrary direction in $\boldsymbol{x}$-space) in the sense that the expressions for a vector displacement $(d \boldsymbol{x})_{\mathcal{K}_{t}}$ of a point of the kink $\mathcal{K}_{t}$ in an infinitesimal 
time interval $d t$, when computed in terms of variables on the two sides of a kink surface, have the same value.

This theorem assures that the 3-D KCL are physically realistic.

\section{Comparison of a KCL Based Method with Level Set Method}

Some important and highly developed methods for tracking the evolution of curves and surfaces are:

Level set method by Sethian deals directly with the equation (1.3) with $\chi$ given by (2.1), i.e.,

$$
\varphi_{t}+m|\nabla \varphi|=0
$$

and discretizing it in $(\boldsymbol{x}, t)$-space, i.e., $(x, y, t)$-space for $d=2$.

Fast marching method by Osher and Sethian, in which we set $\varphi=T(x, y)-t$ and reduce the equation (5.1) to $m|\nabla T|=1$. This reduces the problem in $d+1$ independent variables to a problem in $d$ independent variables as in the case of KCL. Theory of characteristics of this equation also leads to the equations (2.2) and (2.3).

In both methods $m$ can be chosen in many ways, say as a known function of $\boldsymbol{x}$ and $t$ or the curvature of $\Omega_{t}$ etc. These are been discussed in a great detail in literature, specially in the book by Sethian [18].

As seen from our investigations in past (see the work our group listed in references), KCL based methods has been very successful in dealing with nonlinear wave front and shock front in a hyperbolic system. It would be worth considering these problems by LSM and FMM. For example when $\Omega_{t}$ is a weak nonlinear wavefront, we get an additional equation, namely the energy transport equation

$$
m_{t}+m\langle\mathbf{n}, \nabla\rangle m=\Omega(m-1)=-\frac{1}{2}<\nabla, \mathbf{n}>(m-1),
$$

which is the same as the equation (6.3) in [1] but written in $(\boldsymbol{x}, t)$-space. Note that $\Omega=-\frac{1}{2}\langle\nabla, \mathbf{n}\rangle$ is the mean curvature of $\Omega_{t}$. If we wish to find evolution of a weekly nonlinear wavefront by LSM, we need to solve the two equations (5.1) and (5.2) together.

No work has been done in comparing the the level set based methods and KCL based methos on common problems and this will require a major research initiative. However, we need to note that in many applications of KCL, we shall have difficulty due to appearance of singularities as $m \rightarrow 1$ and change in the nature of the system of equations from $m<1$ to $m>1$. 


\section{ACKNOWLEDGEMENT}

This work is supported by National Academy of Sciences, India under NASI Senior Scientist Platinum Jubilee Fellowship programme. The Department of Mathematics of IISc is partially supported by the UGC as a Centre of Advance Study and by DST through FIST Programme.

\section{REFERENCES}

1. K. R. Arun and Phoolan Prasad, 3-D Kinematical Conservation Laws (KCL): Equations of evolution of a surface, Wave Motion, 46 (2009), 293-311.

2. K. R. Arun, M. Lukacova-Medvidova, Phoolan Prasad and S. V. Raghurama Rao, An application of 3-D kinematical conservation laws: Propagation of a three dimensional wavefront, SIAM J. Appl. Math., 70 (2010), 2604-2626.

3. K. R. Arun and Phoolan Prasad, Eigenvalues of kinematical conservation laws (KCL) based 3-D weakly nonlinear ray theory (WNLRT), Applied Mathematics and Computation, 217 (2010), 2285-2288.

4. K. R. Arun, A numerical scheme for three-dimensional front propagation and control of Jordan mode, SIAM J. Sci. Comput., 34 (2012), B148-B178.

5. K. R. Arun and Phoolan Prasad, Propagation of a three-dimensional weak shock front using kinematical conservation laws, item 17, on the webpage of Phoolan Prasad, Reprints and Preprints, http://math.iisc.ac.in/ prasad/.

6. S. Baskar and Phoolan Prasad, Kinematical conservation laws applied to study geometrical shapes of a solitary wave, Wind over Waves II: Forecasting and Fundamentals, Ed. S. Sajjadi and J. Hunt, Horwood Publishing Ltd, 189-200, 2003.

7. S. Baskar and Phoolan Prasad, Riemann problem for kinematical conservation laws and geometrical features of nonlinear wavefronts, IMA Journal of Applied Mathematics, 69 (2004), $391-420$.

8. S. Baskar and Phoolan Prasad, Propagation of curved shock fronts using shock ray theory and comparison with other theories, J. of Fluid Mechanics, 523 (2005), 171-198.

9. S. Baskar and Phoolan Prasad, Formulation of the problem of sonic boom by a maneuvering aerofoil as a one parameter family of Cauchy problems, Proceedings of Indian Academy of Sciences : Mathematical sciences, 116 (2006), 97-119.

10. R. Courant and F. John, Introduction to Calculus and Analysis, Vol II, John Wiley and Sons, New York, 1974.

11. L. C. Evans, Partial Differential Equations, Graduate Studies in Mathematics, Vol 19, American Mathematical Society, 1999. 
12. M. Giles, P. Prasad and R. Ravindran, Conservation form of equations of three dimensional front propagation, Technical Report, Department of Mathematics, Indian Institute of Science, Bangalore, 1995.

13. N. K. R. Kevlahan, The propagation of weak shocks in non-uniform flow, J. Fluid Mech., 327 (1996), 167-97.

14. K. W. Morton, P. Prasad and R. Ravindran, Conservation forms of nonlinear ray equations, Technical Report No.2, Department of Mathematics, Indian Institute of Science, Bangalore, 1992.

15. Phoolan Prasad, Nonlinear Hyperbolic Waves in Multi-dimensions, Monographs and Surveys in Pure and Applied Mathematics, Chapman and Hall/CRC, 121, 2001.

16. Phoolan Prasad, Ray theories for hyperbolic waves, kinematical conservation laws and applications, Indian J. Pure and Appl. Math., 38 (2007), 467-490.

17. R. Ravindran and Phoolan Prasad, On infinite system of compatibility conditions along a shock ray, Q. J. Appl. Math. and Mech., 46 (1993), 131-140.

18. J. A. Sethian, Level set methods and fast marching methods: Evolving surfaces in computational geometry, fluid mechanics, computer vision, and material sciences, Cambridge University Press, second edition, 1999.

19. B. Sturtevant and V. A. Kulkarni, The focusing of weak shock waves, J. Fluid Mech., 73 (1976), 651-71.

20. B. Temple and J. Smoller, Expanding wave solutions of the Einstein equations that induce an anomalous acceleration into the Standard Model of Cosmology, Proc. National Academy of Sciences, USA, 106(34) (2009), 14213-14218.

21. G. B. Whitham, Linear and Nonlinear Waves, John Wiley \& Sons, New York, 1974. 